\title{
Study on Detailed Calculation and Experiment Methods of Neutronics, Fuel Materials, and Thermal Hydraulics for a Commercial Type Japanese Sodium-Cooled Fast Reactor
}

\author{
Toshikazu Takeda, W. F. G. van Rooijen, Katsuhisa Yamaguchi, Masayoshi Uno, \\ Yuji Arita, and Hiroyasu Mochizuki
}

Research Institute of Nuclear Engineering, University of Fukui, 1-2-4 Kanawa-cho, Tsuruga-shi, Fukui 914-0055, Japan

Correspondence should be addressed to Toshikazu Takeda, t_takeda@u-fukui.ac.jp

Received 4 November 2011; Accepted 12 January 2012

Academic Editor: Massimo Salvatores

Copyright (C) 2012 Toshikazu Takeda et al. This is an open access article distributed under the Creative Commons Attribution License, which permits unrestricted use, distribution, and reproduction in any medium, provided the original work is properly cited.

\begin{abstract}
This paper discusses the objectives and results of a multiyear R\&D project to improve the modeling accuracy for the detailed calculation of the Japanese Sodium-cooled Fast Reactor (JSFR), although the preliminary design of JSFR is prepared using conventional methods. For detailed design calculations, new methods are required because the JSFR has special features, which cannot be accurately modeled with existing codes. An example is the presence of an inner duct in the fuel assemblies. Therefore, we have developed new calculational and experimental methods in three areas: (1) for neutronics, we discuss the development of methods and codes to model advanced FBR fuel subassemblies, (2) for fuel materials, modeling and measurement of the thermal conductivity of annular fuel is discussed, and (3) for thermal hydraulics, we describe advances in modeling and calculational models for the intermediate heat exchanger and the calculational treatment of thermal stratification in the hot plenum of an FBR under low flow conditions. The new methods are discussed and the verification tests are described. In the validation test, measured data from the prototype FBR Monju is partly used.
\end{abstract}

\section{Introduction}

Many scenarios of possible energy futures foresee an important role for nuclear power. Acceptance of nuclear power with large-scale contributions to the world's energy mix depends on satisfaction of key drivers to enhance sustainability in terms of economy, safety, adequacy of natural resources, waste reduction, and nonproliferation. Fast reactors with recycle significantly enhance the sustainability indices. Therefore, due to their flexibility to produce new fissile material and/or to reduce the amount of waste and its impact on the environment, fast reactors are needed to make nuclear power a truly long term option.

Japan has a long track record of maintaining a commitment to fast reactor technology. Considerable efforts have been focused on innovative design approaches to achieve optimal safety, waste minimization, enhanced proliferation resistance, and economical competiveness. Studies carried out over the past decade $[1,2]$ have concluded that these objectives could be achieved. All types of fast reactor designs were considered, including advanced loop and pool configurations, various fuel types and coolants, and complete costs over the entire life cycle. The system chosen in Japan for detailed design is a sodium-cooled fast reactor known as the Japan Sodium-cooled Fast Reactor (JSFR), a two-loop, oxide-fueled system rated at an electrical output of 1,500 MW [3-5]. In Figures 1 and 2 are illustrated a 3D view of the plant arrangement and a schematic of the primary and secondary sodium loops. In this JSFR, technical innovations such as a large fuel assembly using large-diameter fuel pins stacked with annular pellets, an inner duct to discharge molten fuel during a hypothetical core destructive accident, and a tall steam generator using straight, double-wall heat transfer tubes are adopted [6].

Technological innovation in the fields of safety and reliability is the key for commercialization of fast reactor for 


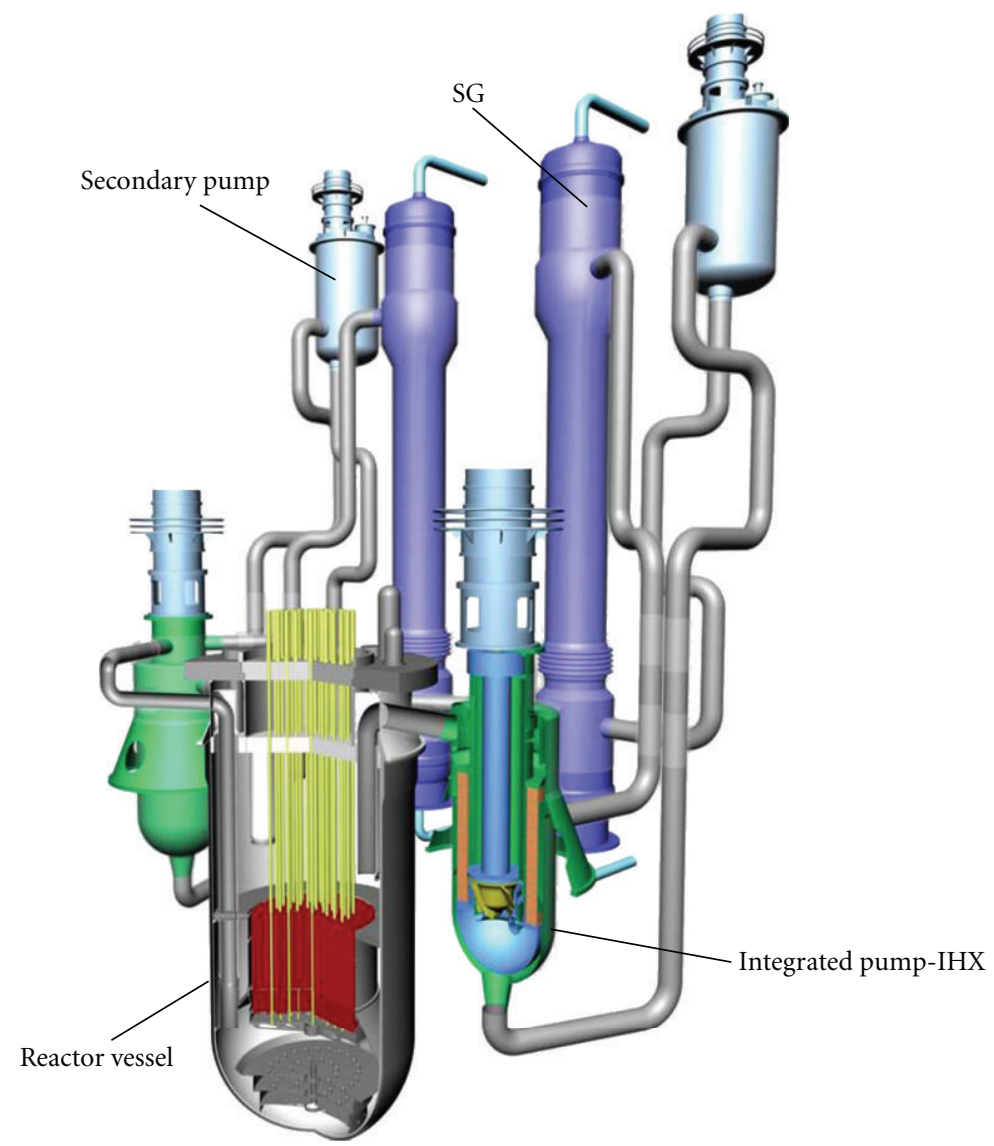

FIGURE 1: Schematic of JSFR-1500, showing the 2-loop arrangement with straight-tube heat exchangers, and with the primary pumps integrated into the Intermediate Heat Exchanger (IHX) (reproduced from [7]).

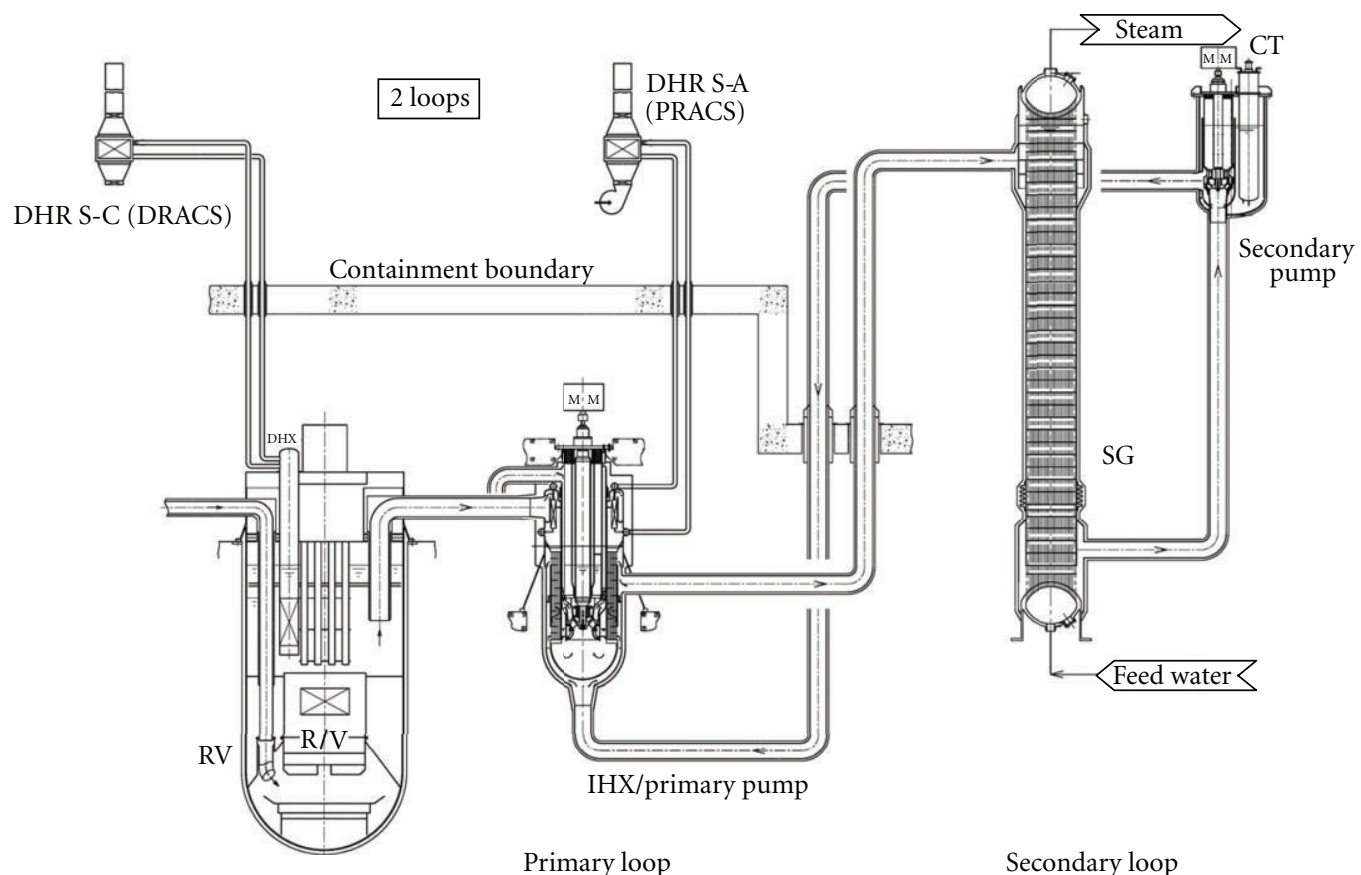

FIgURE 2: Primary and secondary circuits of JSFR-1500 (only 1 of 2 loops shown) (reproduced from [7]). 
sustainable development. It is pursued in a wide range of scientific and technical area, including nuclear data, reactor physics, engineering design, and methods of validation and qualification.

Monju will produce a wealth of physics and performance as well as operational experience data. Efforts that make use of these data and provide the feedback needed to improve the designs of future fast reactors are a must. In particular, data and computer code Verification, Validation, and Uncertainty Qualification (V\&V, UQ) with the help of theoretical and experimental studies will be most beneficial. In this paper, therefore, the development of calculation and experiment methods relevant to the reactor physics, fuel, and thermal hydraulics is described. Main focus is the application of the result to the design of an advanced commercial type sodiumcooled fast reactor.

To design the FBRs of the future, it is a requirement that one is able to calculate the physical characteristics accurately, that is, without the need of mock-up experiments. This requires advanced modeling tools. High-priority issues are the modeling of neutronics, fuel and structural materials, and thermal-hydraulics. In the present paper, these fields are introduced independently. However, in the future the models will be coupled to properly analyze and evaluate the overall behavior of the plant.

In the area of neutronics, the application of transport theory for full-core calculations requires accurate treatment of highly heterogeneous subassemblies. Therefore we are focusing on improvements of cell calculations: a hyperfine group treatment allows to explicitly treat all effects of crosssection resonances, while the use of advanced transport theory methods allows to explicitly treat highly heterogeneous subassemblies. In the area of fuel materials, we are focusing on the accurate modeling of advanced fuels in which particles are dispersed to improve material properties, especially the thermal conductivity. Furthermore, we are developing measurement technology for the pre- and postirradiation analysis of fuel pellets. Finally, in the area of thermal-hydraulics we are targeting several long-standing issues, that is, the poorly understood degradation of heat exchange in the Intermediate Heat Exchanger under low flow conditions, as well as the accurate $3 \mathrm{D}$ simulation of the temperature field in the hot plenum above the core from the standpoint of maintenance, reliability, component lifetime, and safety.

\section{Neutronics Calculation Methods}

The calculation methods for the commercial type Fast Breeder Reactor (FBR) have been developed based on deterministic methods. As the nuclear data we selected the JENDL-4.0 nuclear data set (Japan Evaluated Nuclear Data Library v4.0) since the core performance parameters of various fast reactor cores and critical assemblies calculated with JENDL-4.0 are closer to the measured data than the results of JENDL-3.3. Figure 3 shows the ratio of calculation to measurement $(\mathrm{C} / \mathrm{E}$ ratio) of $k_{\text {eff }}$ of several fast systems, comparing JENDL-3.3 and JENDL-4.0. In particular, JENDL-4.0 improves the underestimation of $k_{\text {eff }}$ for uranium-fueled cores compared to

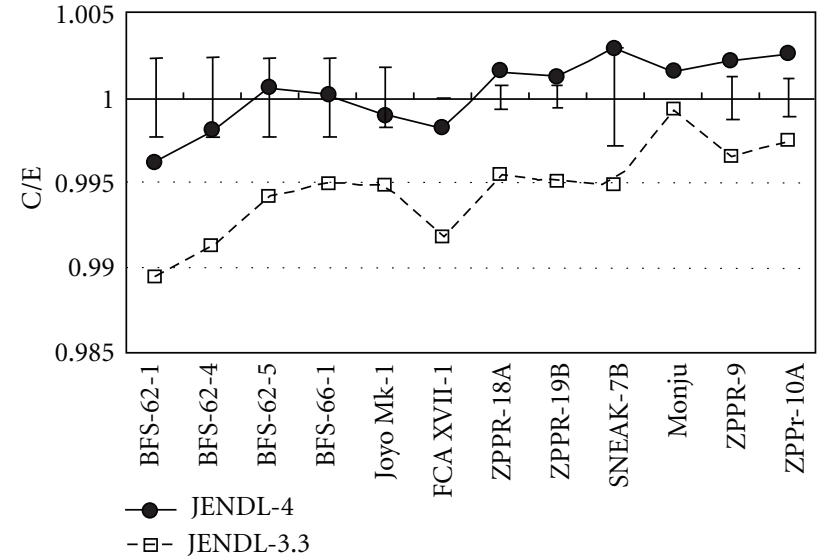

Figure 3: Comparison of the C/E ratio for $k_{\text {eff }}$, using JENDL3.3 and JENDL-4.0 for several fast systems (reproduced from [8]). Vertical bars indicate experimental uncertainties. BFS: critical facility at IPPE (Russia, [9]); FCA: Fast Critical Assembly at JAEA Tokai; ZPPR: Zero Power Plutonium Reactor at Idaho National Lab, SNEAK: Schnelle Null-Energie-Anordnung Karlsruhe (Fast ZeroPower Facility Karlsruhe, Germany).

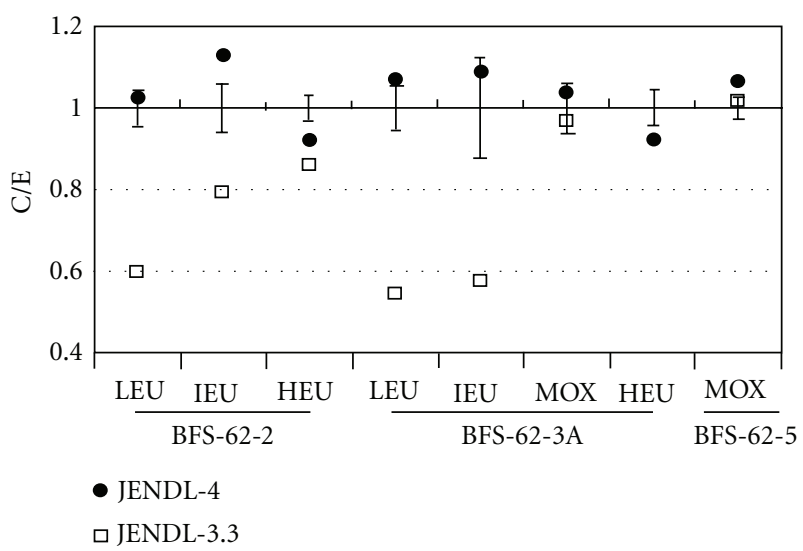

Figure 4: Prediction of SVR with JENDL-3.3 and JENDL-4.0 for several configurations of the BFS facility in IPPE (Russia [9]) (Reproduced from [8]). Vertical bars represent experimental uncertainties. HEU/IEU/LEU: High/Intermediate/Low Enriched Uranium; MOX: Mixed Oxide, that is, uranium-plutonium oxide fuel.

JENDL-3.3 (results of the BFS facility in Figure 3). Figure 4 shows the improvement of the prediction of the Sodium Void Reactivity (SVR) by JENDL-4.0 compared to JENDL-3.3 for several uranium-fueled configurations of the BFS facility. The prediction of the SVR is considerably improved. For other critical assemblies (e.g., ZPPR and SEFOR), a small improvement was found.

To obtain effective groupwise cross sections, the SLAROM-UF code has been selected. (SLAROM is a lattice analysis code developed by JAEA; UF stands for UltraFine.) Traditionally in Japan, effective cross-sections are calculated in 70 energy groups with the SLAROM and SRAC codes, using equivalence theory with a Dancoff factor (Standard Reactor Analysis Code, a modular code system for thermal reactor 


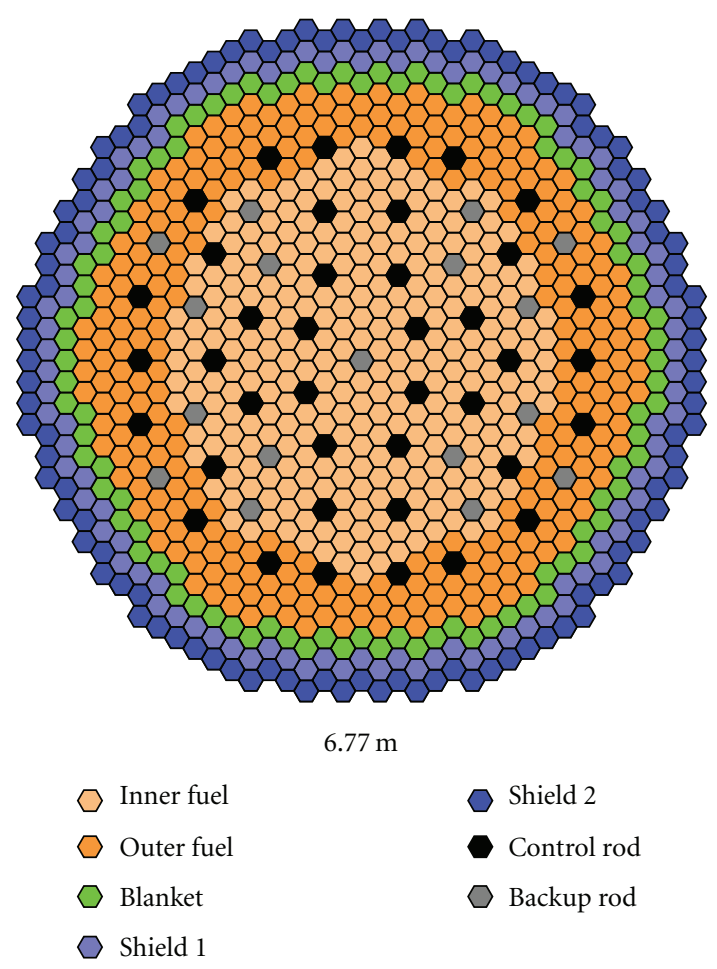

Figure 5: Core map of JSFR-1500 [6]. analysis developed by JAEA). In this kind of calculation, the problem-specific composition and geometry can only be taken into account partially, because the group cross-sections are calculated with a fixed neutron energy spectrum (weight flux) rather than a full problem-dependent neutron energy spectrum. Mutual self-shielding effects are also neglected.

The SLAROM-UF code has been developed by JAERI [10, 11] to treat ultrafine energy groups, using up to a hundred thousand groups below $50 \mathrm{keV}$. (JAERI: Japan Atomic Energy Research Institute, one of the predecessors of the present JAEA, Japan Atomic Energy Agency). The use of ultrafine energy groups leads to an improvement not only for the criticality calculations but also for the SVR $[9,12]$.

The commercial-size JSFR is a 1,500 MWe fast reactor with a core of $6.77 \mathrm{~m}$ diameter and $1.5 \mathrm{~m}$ height, as shown in Figures 5 and 6 . In the case of a severe accident in a traditionally designed core the molten fuel may accumulate in some part of the core, and cause a recriticality. To prevent the occurrence of recriticality, there is an inner duct installed in each fuel assembly. In case of accidents, the molten fuel flows upward through the inner duct, and a recriticality is prevented.

Thus the JSFR fuel assembly has a complicated form, and the accurate description of the assembly should be taken into account when calculating assembly averaged cross-sections. To calculate the homogenized cross-sections, the Method of Characteristics (MOC) has been used because the complex geometry can be taken into account accurately. The effects of heterogeneities on the assembly-averaged cross-sections are taken into account by the MOC calculation.

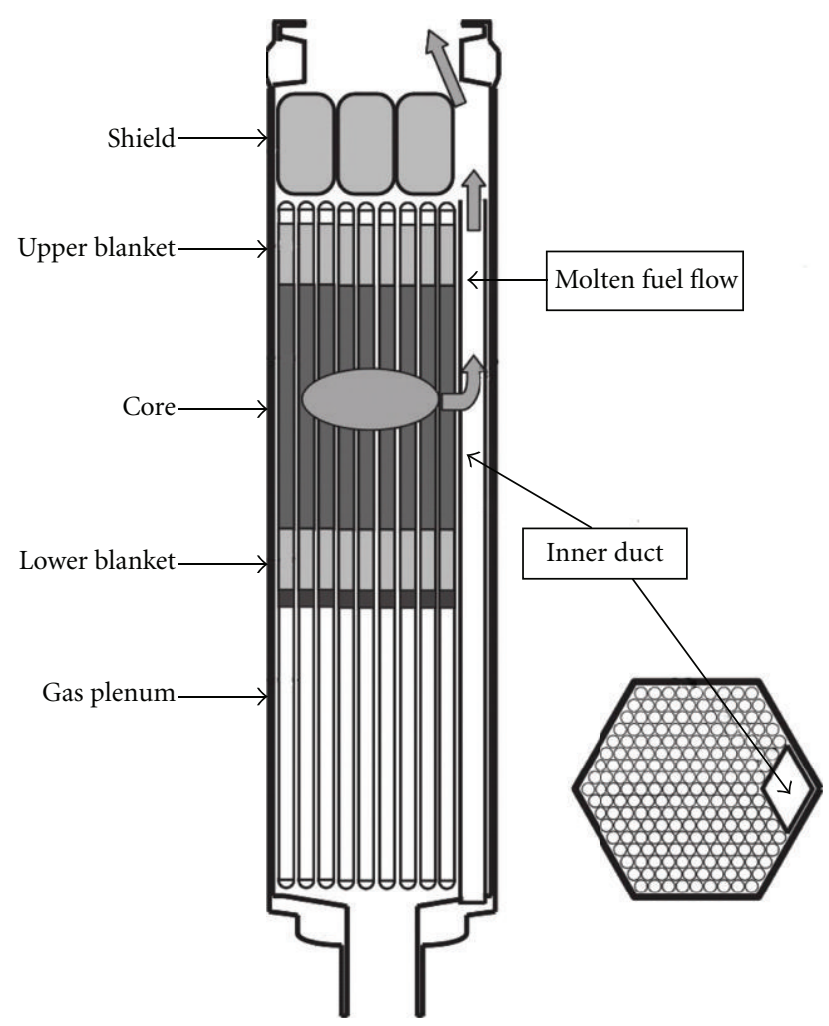

FIGURE 6: JSFR fuel assembly with internal duct (so-called modified FAIDUS concept, FAIDUS: Fuel Assembly with Inner DUct Structure) [13].

For ultrafine group calculations, the modeling options in SLAROM-UF are limited to one spatial dimension (1D): the presence of the inner duct cannot be treated. For this reason, it was chosen to incorporate the $\mathrm{BACH}$ code $(\mathrm{Be}-$ neficial Advanced Characteristics code for Hexagonal geometry [14]), a 2D MOC code for hexagonal geometry. In the new system, SLAROM-UF acts as a cross-section production module, producing effective cross-sections in a 70-group structure, while $\mathrm{BACH}$ is used to determine an accurate flux distribution and cross sections to be used in the core calculations (see Figure 7; more information about the codes mentioned in the figure is given later).

In order to verify the accuracy of the BACH code, we have compared the $k_{\text {eff }}$ values and the intra-assembly reaction rate distributions. Figures 8 and 9 compare the U-238 capture rate distributions, calculated by the $\mathrm{BACH}$ code and the continuous-energy Monte-Carlo code MVP, in a fuel assembly of Monju and in a JSFR assembly, respectively. Good agreement is found for both assemblies, which shows the accuracy of the $\mathrm{BACH}$ code. In Figure 8, the fuel pins have been grouped into three groups labeled A, B, and C. This grouping is introduced to take into account the differences in self-shielding. The influence of the wrapper tube and the interassembly sodium is small for pins in group A, but larger for pins in groups $\mathrm{B}$ and $\mathrm{C}$. Therefore, three sets of multigroup cross-sections were calculated representing three different levels of selfshielding. 


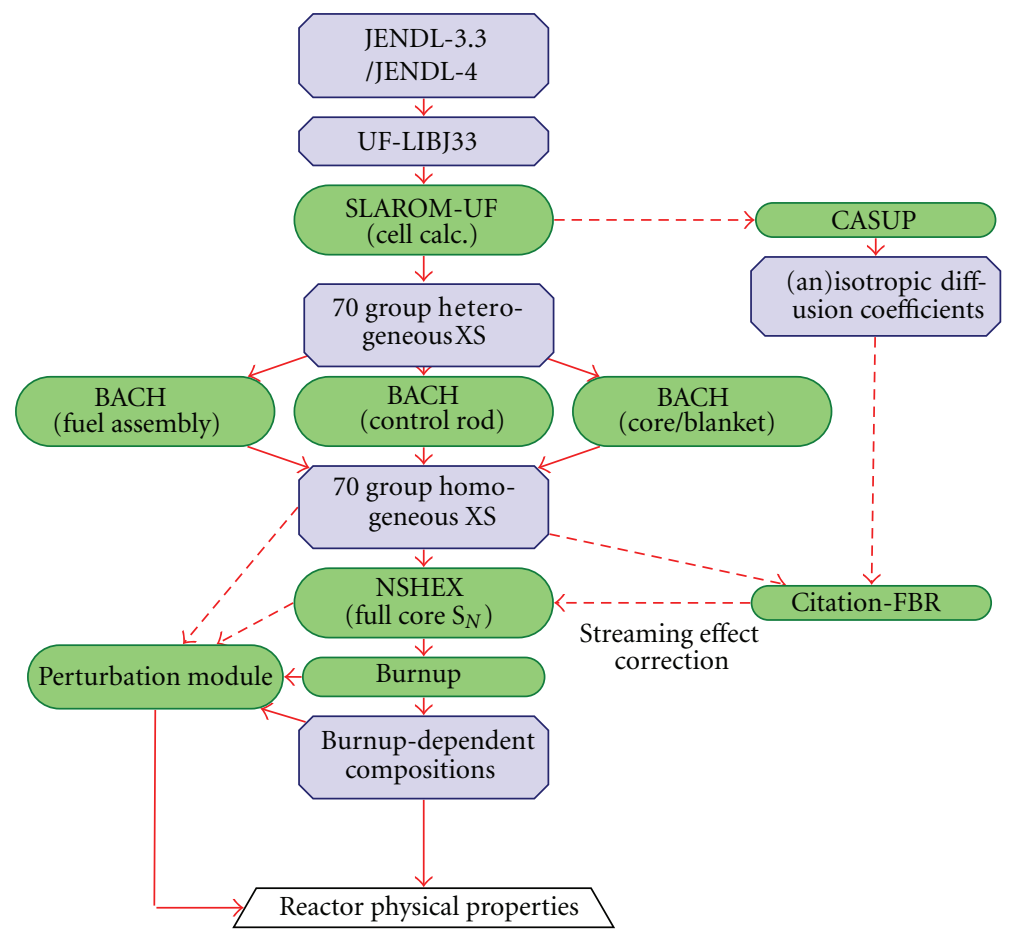

FIGURE 7: Flow chart of a reactor physics calculation.
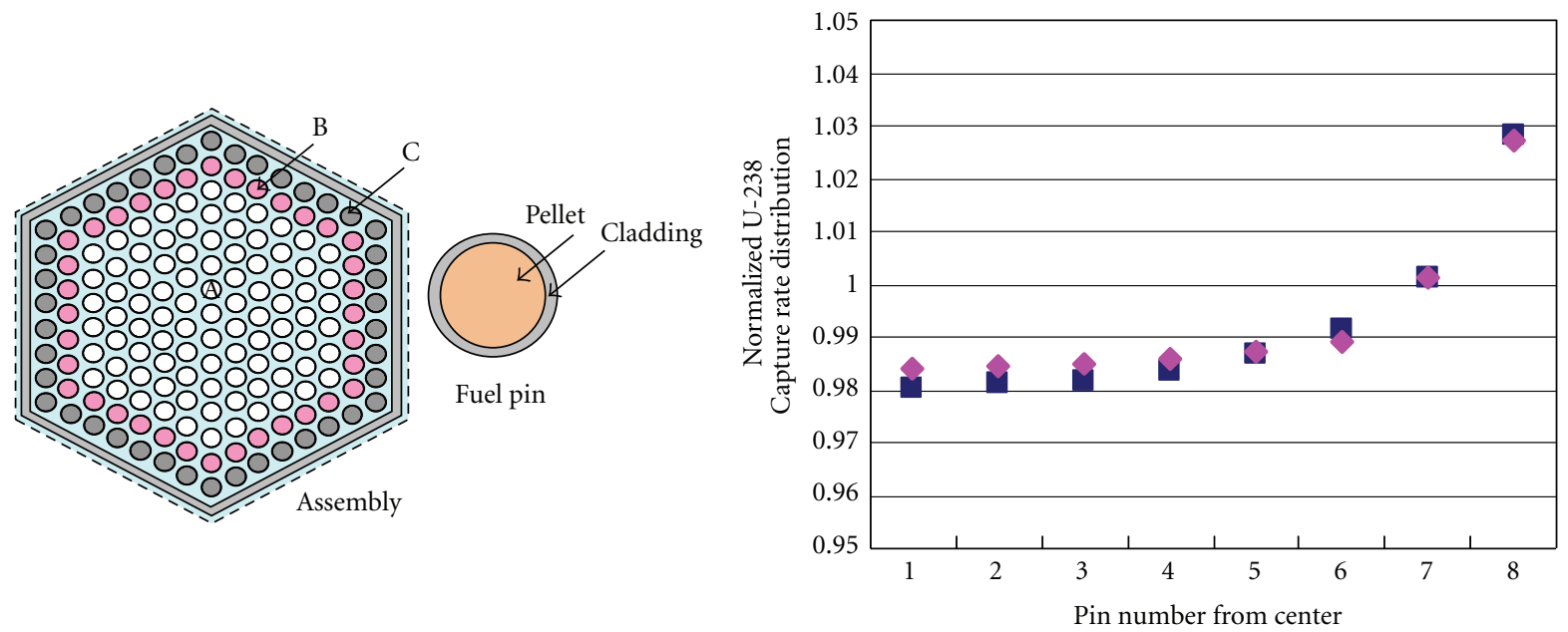

- MVP

$\mathrm{BACH}$

Figure 8: U-238 Capture Rate Distribution in a Monju fuel assembly. Fuel pins are grouped into three groups (A, B, and C) to take into account the difference in self-shielding between fully surrounded fuel pins and fuel pins near the wrapper tube and interassembly sodium channel.

By default, assembly averaged cross sections are calculated assuming an infinite lattice of repeating assemblies. For special assemblies, such as control rod assemblies, color set calculations are used, as shown in Figure 10.

After the preparation of homogenized cross sections, core calculations are performed with transport theory. The code NSHEX is a nodal Sn transport code, described in more detail in [15]. It is capable of treating Hexagonal-Z geometry with several options for the nodal expansion order and Sn quadrature sets. In this figure the diffusion code CITATIONFBR also appears. (An improved version for FBR applications of the traditional diffusion code CITATION; CITATIONFBR is developed by JAEA). This code is used in triangular- $Z$ geometry. CITATION-FBR is capable of using anisotropic diffusion coefficients. This allows one to take into account effects of anisotropy in the case of a voided lattice, and so forth. 

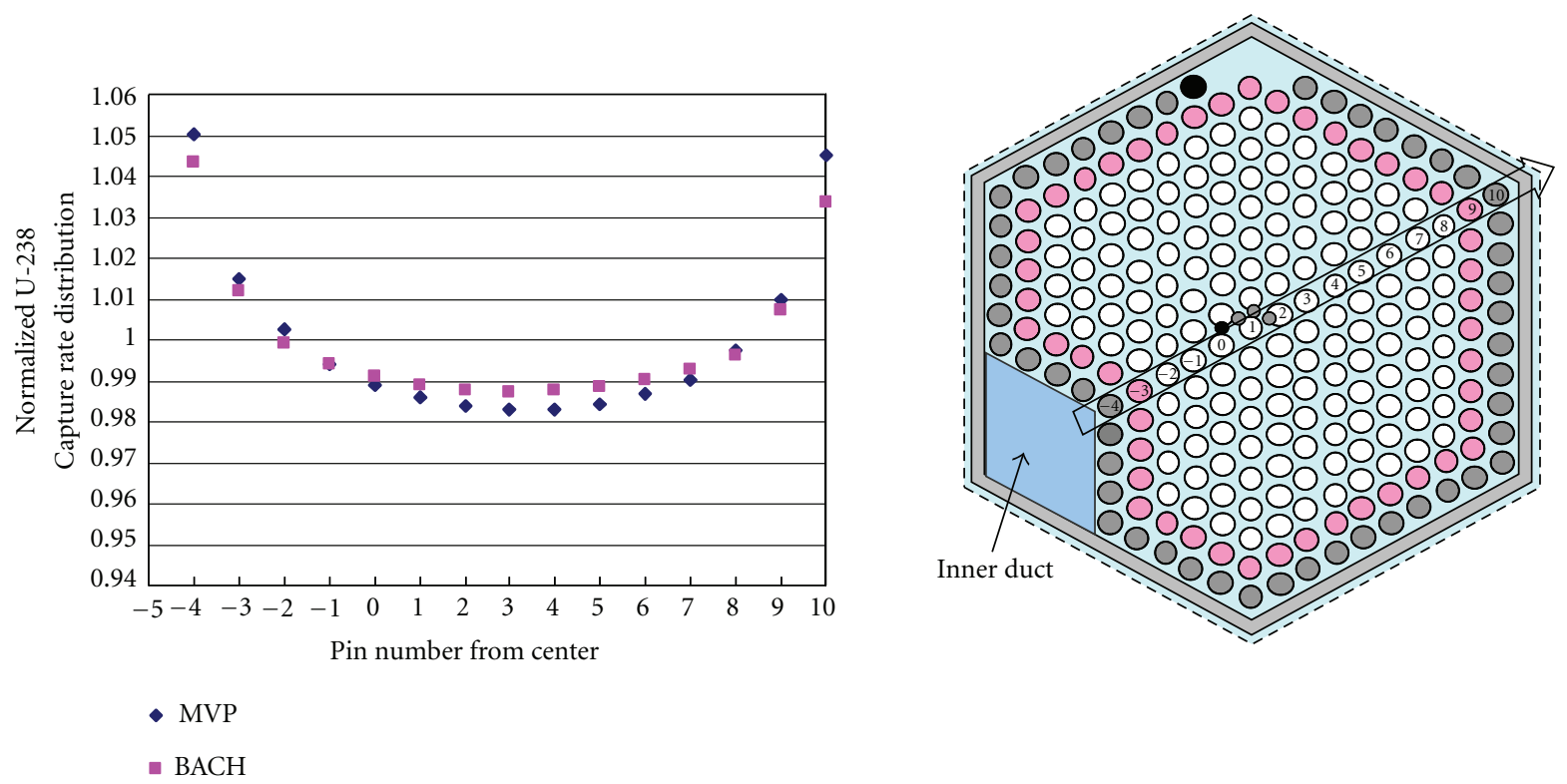

Figure 9: U-238 Capture Rate Distribution in a JSFR fuel assembly. Fuel assemblies are grouped into three groups to take into account the influence of the wrapper tube and sodium channels on the self-shielding.

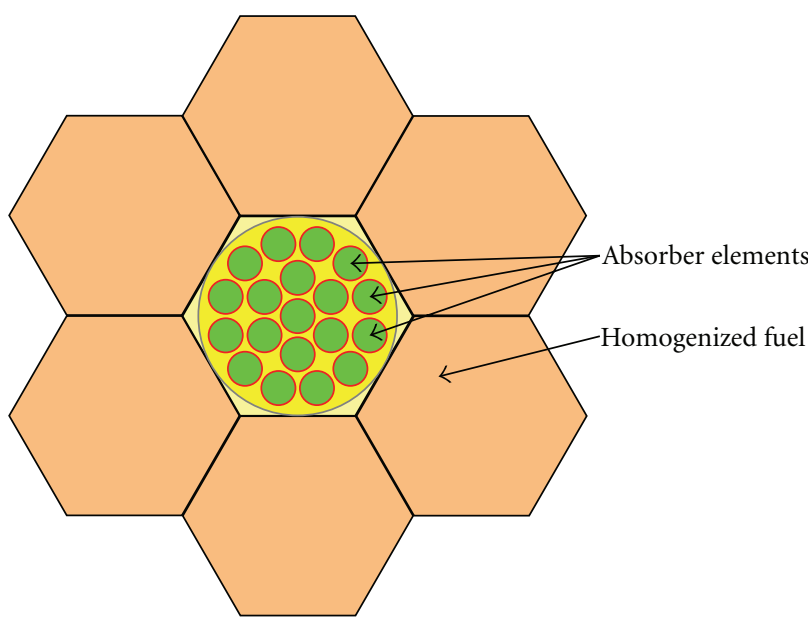

Figure 10: Color set calculation.

The code NSHEX can only treat isotropic scatter, while of course the angular flux in NSHEX can be anisotropic.

\section{Calculation and Experiment Methods for Fuel}

3.1. Calculation of the Thermal Conductivity of Particle Dispersing Fuels. The thermal conductivity of nuclear fuels is one of the most important properties concerning reactor safety. The Japanese code CEDAR [16] estimates the behavior of fast reactor fuels during irradiation using the thermal conductivity in addition to other material properties. In fast reactors, the centerline temperature of MOX fuel pellets increases up to the melting temperature of the MOX and a hole appears in the central part of the pellet early in the irradiation period. This is caused by the high heat flux and low thermal conductivity of the MOX fuel matrix [1719]. The high heat flux combined with a steep temperature gradient has other effects, including the formation of cracks, redistribution of Fission Product (FP) and Trans-Uranium (TRU) atoms, the formation of precipitates of fuel and FP atoms in the middle part of the pellet, and the formation of a so-called Joint Oxide Gaine (JOG) at the pellet-clad interface. To improve the thermal conductivity, it has been proposed to disperse particles with a high thermal conductivity in the MOX matrix. In a previous study a $\mathrm{Gd}_{2} \mathrm{O}_{3}$-dispersed $\mathrm{UO}_{2}$ fuel was proposed to improve the thermal conductivity of $\mathrm{UO}_{2}$ pellets [20]. LWR fuel additives have many restrictions from the viewpoint of the capture of thermal neutrons; however, for FRs there are less limitations concerning additives, since the capture cross section of the concerned isotopes usually decreases with increasing neutron energy. In the present study, we investigated dispersion parameters such as particle size, shape, and concentration, in an effort to improve the thermal conductivity of the MOX fuel pellet in the early stages of irradiation. The particles are assumed to be randomly distributed. The effect of the dispersed particles after long irradiation is being investigated.

3.1.1. Calculation Procedure. The radial temperature distributions in pellets with dispersed particles were calculated using a FEM-based approach. The general FEM analysis for temperature distributions requires the solution of thermal transfer equations by considering several factors such as thermal diffusivity, heat capacity, material density, and heat generation [21].

In this study, systems containing distributed spherical particles, needle-like particles, and flat particles within the matrix were considered (see Figure 11). The MOX pellets are $10 \mathrm{~mm}$ in diameter and $10 \mathrm{~mm}$ in height. The dispersed 


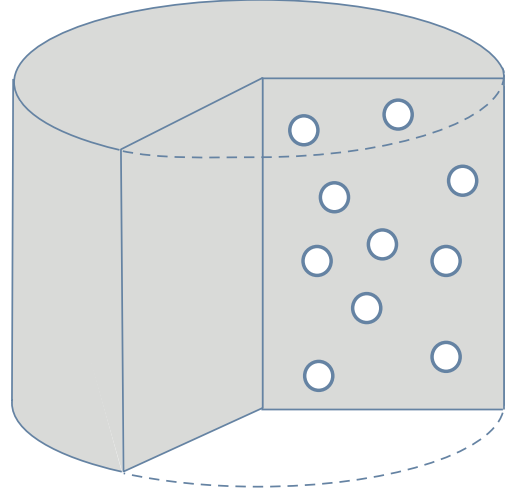

(a)

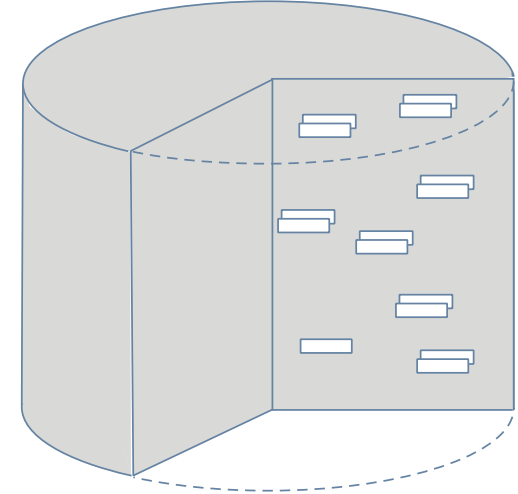

(b)

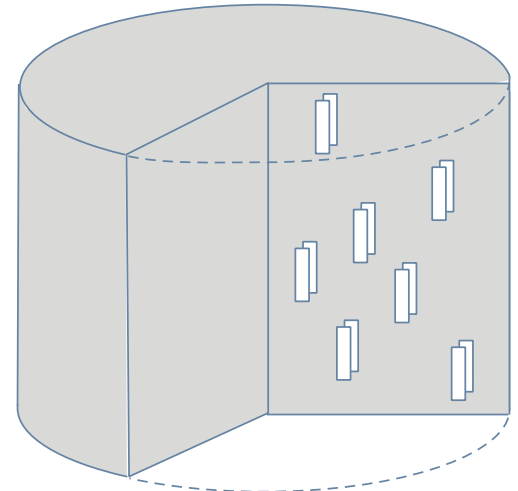

(c)

FIgURE 11: Dispersed particle shapes in the pellet matrix: (a) spherical balls, (b) needles, and (c) flat plates.

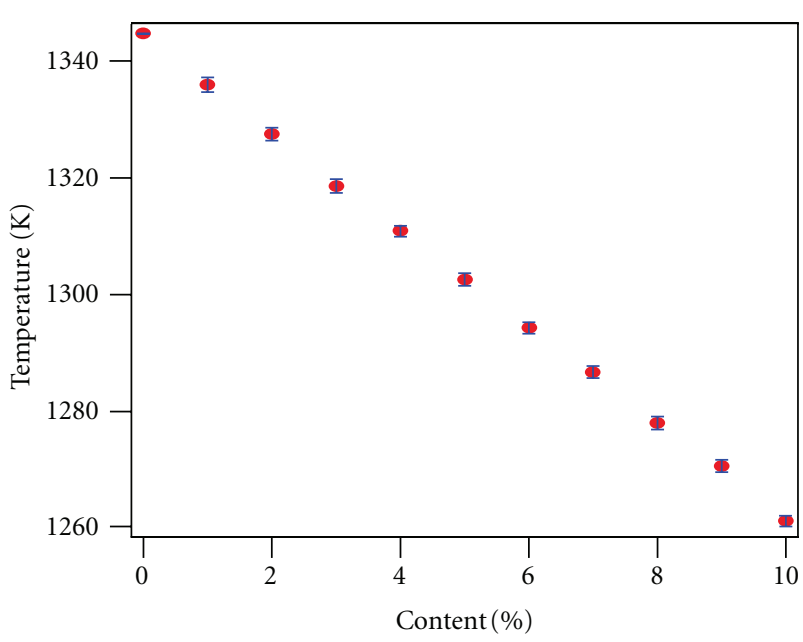

FIGURE 12: Centerline temperature as a function of particle content (spherical particles, $100 \mu \mathrm{m}$ in diameter).

particles are spherical balls of $30-500 \mu \mathrm{m}$ in diameter, needles of $100 \times 100 \times 500 \mu \mathrm{m}$ in dimension, and flat plates of $500 \times 500 \times 100 \mu \mathrm{m}$. The heat source for the MOX matrix and the external temperature of the pellet was fixed for the calculations. In the present study it is assumed that the thermal conductivity of the dispersed particles is ten times higher than that of the MOX matrix [17-19].

3.1.2. Results and Discussion. For the spherical particles the dependence of the pellet centerline temperature on the concentration of the particles was analyzed. Figure 12 shows the centerline temperature for pellets with a particle content ranging from 0 to $10 \mathrm{vol} \%$. The centerline temperature decreases linearly with particle content. From this result the centerline temperature is effectively decreased by increasing the amount of dispersed particles. The amount to be used will be determined by the required performance of the fuel pellet.

The dependence of the centerline temperature on the size of the particles was investigated using a dispersion of spherical balls with diameters ranging from 30 to $500 \mu \mathrm{m}$. The

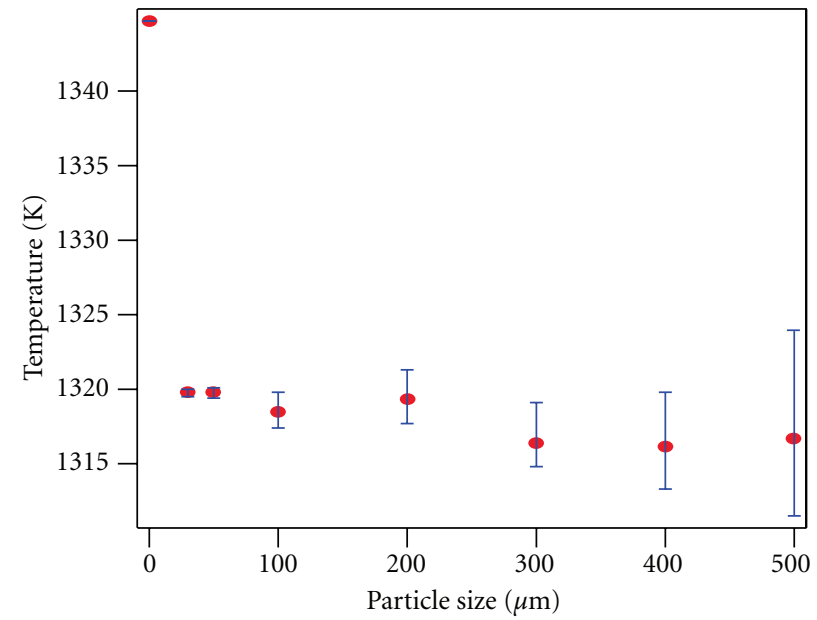

Figure 13: Centerline temperature as a function of the diameter of the spherical particles ( 3 vol\% content).

result is illustrated in Figure 13, which shows the results for a total amount of dispersed particles of $3 \mathrm{vol} \%$. At this volume fraction of particles, the pellet centerline temperature is more or less constant as a function of the size of the particles. This means that the contribution to the overall heat transfer of the pellet is determined by the volume fraction of the dispersed particles. However, the temperature distribution becomes less smooth as the diameter of a particle increases. As seen in Figure 14, the region near the particles has a low temperature and the area far from particles has a high temperature. Therefore, to minimize thermal stress and retain the integrity of the pellet, the size of dispersed particles must not be too large and $100 \mu \mathrm{m}$ or less is recommended.

The centerline temperatures of pellets with dispersed spherical balls, needles, or flat plates were calculated. The differences in the centerline temperature upon the addition of spherical balls, needles, and plates were small. For the formation of particles, spherical dispersed particles are recommended. 


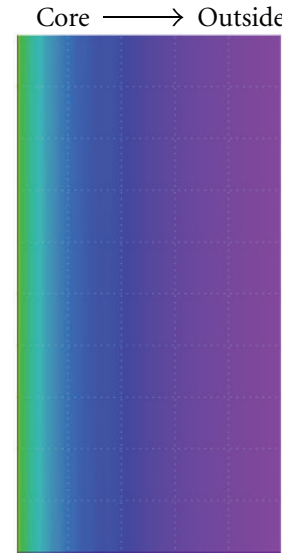

MOX

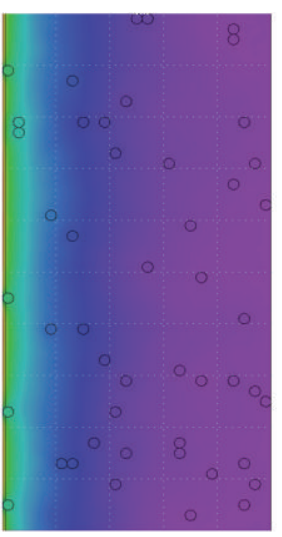

$200 \mu \mathrm{m}$

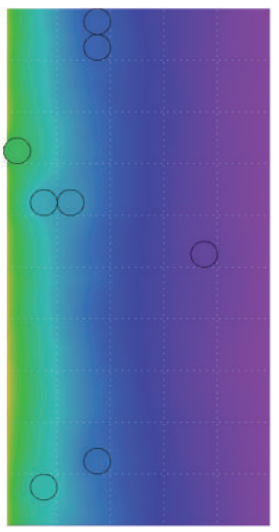

$500 \mu \mathrm{m}$

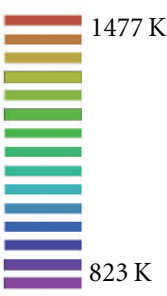

FIGURE 14: Temperature distribution inside a pellet cross with different sizes of the spherical dispersed particles (3 vol\% content).

\subsection{Measurement of the Thermal Conductivity of the Annular} Pellet. The current fast reactor fuel pellet has a low smear density but the pellets to be used in a demonstration reactor will be annular with a high density. It is difficult to measure the thermal conductivity of an annular pellet with a thermal diffusivity measurement with a conventional laser flash method. The method is especially problematic in a hot cell, since it requires a disk-shaped sample with a surface cross section of 1 or $2 \mathrm{~cm}^{2}$ and a thickness of about 1 or $2 \mathrm{~mm}$. It is very difficult to prepare such samples for measurement from irradiated annular pellets. In the present study, the thermal conductivity of an annular pellet was measured using the hot disk method.

The hot disk method is one of the transient plane source methods developed by Gustavsson [21]. In this method, a flat sensor is placed between the plane surfaces of two measurement samples. The sensor is made of Kapton and uses embedded double spiral wires made of Ni. One of the wires is used to heat the sample and the other is used to measure the temperature of the sample. The temperature change is inferred from the change of the electrical resistance of one of the Ni wires, while the other wire provides a constant power supply. By analyzing the temperature change, the values of thermal conductivity and the heat capacity of the samples are obtained simultaneously. The merit of this method is that it provides a rapid and simultaneous measurement. The demerit is that it requires two samples, and the difficulty of high temperature measurements caused by the instability of the coated materials of the sensor. Sensors for liquid samples and large solid samples with hypothetically infinite axial dimensions have been developed, but a sensor for annular pellets has not yet been developed. The research presented here targets the development of a hot disk sensor for annular pellets.

3.2.1. Experimental Method. Simulated annular pellets of $\mathrm{CeO}_{2}$ with an outside diameter of $8 \mathrm{~mm}$ and a central hole with a diameter of $2 \mathrm{~mm}$ were made using the SPS (Spark Plasma Sintering) method. After the SPS step, the sample was heated in air at $1873 \mathrm{~K}$ for 20 hours in order to compensate the reduction during the SPS step. Solid $\mathrm{CeO}_{2}$ pellets were also made, and their thermal conductivity was measured using the hot disk method using a conventional sensor. For comparison, measurements were also made using the laser flash method. In the laser flash method, the thermal diffusivity of the sample was measured with the laser flash apparatus and the thermal conductivity was estimated from the following:

$$
\lambda=\alpha \cdot C_{P} \cdot \rho,
$$

where $\lambda$ is the thermal conductivity, $\alpha$ is the thermal diffusivity, $C_{p}$ is the heat capacity, and $\rho$ is the density.

A hot disk sensor for annular pellets was developed. The sensor has two spiral Ni wires of $7.5 \mathrm{~mm}$ diameter with a central hole of $2 \mathrm{~mm}$ diameter as shown in Figure 15(a). After the surface of the samples was polished, the sensor was placed between two $\mathrm{CeO}_{2}$ samples during the measurement as shown in Figure 15(b).

3.2.2. Results and Discussion. Table 1 shows the thermal conductivity of the samples measured with various methods as well as the literature value for comparison [22]. Since the thermal conductivity depends on the porosity of the sample, the measured values were normalized to those for $100 \%$ theoretical density (TD) using the Maxwell-Eucken equation [23]:

$$
\lambda_{P}=\lambda_{0}(1 \quad P) /(1+P \beta), \quad \beta=0.5,
$$

where $\lambda_{p}$ is the thermal conductivity of porosity $P(=1-$ $I / 100)$, and $\lambda_{0}$ is the thermal conductivity of the material at $100 \%$ TD.

Although all normalized values are a little lower than the literature values, the value of the thermal conductivity of the annular pellet obtained by the hot disk method using the annular sensor agrees well with those of normal pellets obtained by the hot disk method using a conventional sensor and by the laser flash method. This agreement shows the accuracy of 


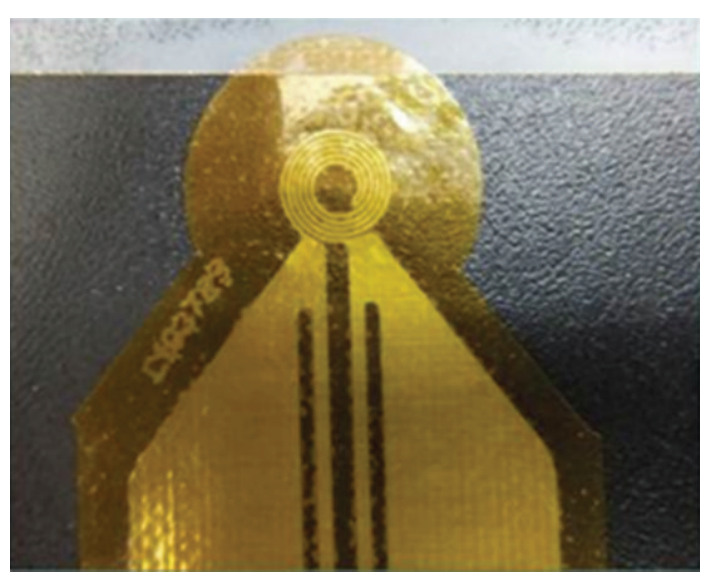

(a)

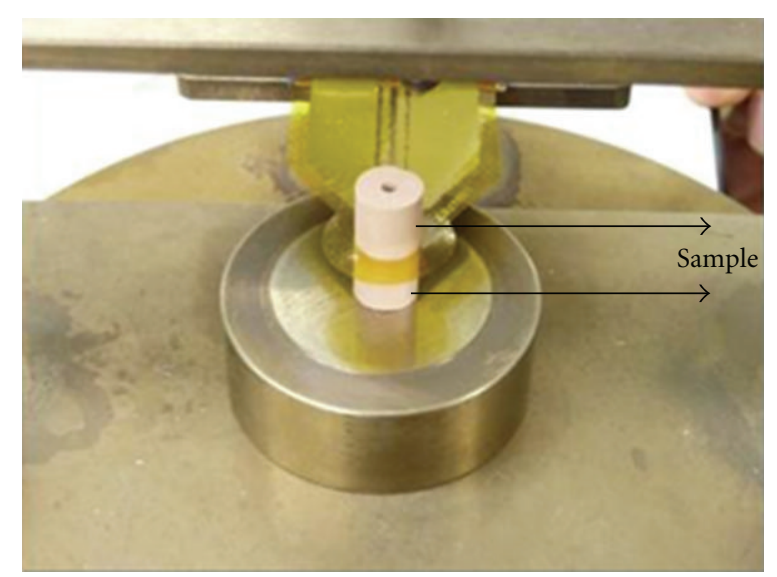

(b)

Figure 15: (a) Hot disk sensor for annular pellets and (b) measurement of the $\mathrm{CeO}_{2}$ annular pellets with the sensor.

TABLe 1: Thermal conductivity of $\mathrm{CeO}_{2}$ pellets measured with several measurement methods.

\begin{tabular}{|c|c|c|c|c|}
\hline $\begin{array}{l}\text { Sample configuration } \\
\text { Density method }\end{array}$ & $\begin{array}{c}\text { Annular } 89 \% \text { TD hot disk } \\
\text { method with the annular } \\
\text { sensor }\end{array}$ & $\begin{array}{l}\text { Normal } 83 \% \text { TD hot disk } \\
\text { method with the normal } \\
\text { sensor }\end{array}$ & $\begin{array}{c}\text { Normal } 83 \% \text { TD laser flash } \\
\text { method }\end{array}$ & Literature value (100\%TD) \\
\hline $\begin{array}{l}\text { Thermal conductivity } \\
(\mathrm{W} / \mathrm{mK}) \text { (as } \\
\text { measured) }\end{array}$ & 9.60 & 8.66 & 8.60 & \\
\hline $\begin{array}{l}\text { Thermal conductivity } \\
\text { (W/mK) (normalized } \\
\text { to } 100 \% \mathrm{TD} \text { ) }\end{array}$ & 11.4 & 11.3 & 11.2 & 12.6 \\
\hline
\end{tabular}

the hot disk method with an annular sensor. As this method does not require cutting of the pellets, this method can be applied to the measurement of irradiated annular pellets in a hot cell.

\section{Thermal Hydraulics}

In order to apply the state-of-the-art technology in the area of thermal hydraulics to the design of the next generation reactor, thermal hydraulic calculations with CFD codes (Computational Fluid Dynamics) are used from the standpoint of V\&V with Monju data; especially, calculations inside of the Intermediate Heat Exchanger (IHX) and the thermal stratification in the upper plenum are targeted. These are common key issues for the design of large sodium-cooled reactors. Since the IHX has a complex configuration with thousands of heat transfer tubes, the precise $3 \mathrm{D}$ calculation is very difficult. Heat exchange in the IHX under low flow conditions has been found to be difficult to predict. Thermal stratification should also be evaluated under low flow rate conditions (e.g., natural circulation).

4.1. Modeling of the IHX. The IHX of Monju is illustrated in Figure 16. The primary sodium enters the shell from a nozzle about halfway up the axial length of the IHX and flows upward in an annular space between the shell and the inner shroud. One flow window is provided in each 60-degree sector. High-temperature sodium should be distributed equally to these windows. For this reason, a flow distribution mechanism consisting of many small holes is provided about half-way up between the nozzle and the flow windows, as illustrated in Figure 17. The flow rate distribution through the different window positions is shown in Figure 18. Because of the distribution of the flow holes over the annulus, the flow distribution is almost uniform over a wide range of flow rates. After entering through the flow window, the sodium flows downward exchanging heat between the primary and the secondary side. There are more than 3000 heat transfer tubes, supported by 7 rectifying plates and 2 tube sheets. Therefore, there are 8 "steps" of the heat transfer regions in the IHX. The rectifying plate has holes through which the heat transfer tubes pass, as well as holes for the sodium flow.

Because of the aforementioned configuration, a CFD mesh of the whole IHX is very difficult. Therefore, a $1 / 6 \mathrm{sec}-$ tor model is discussed. The whole shell and two of the heat transfer regions are modeled based on the design. Calculations are performed to confirm the flow distribution in the heat transfer regions. The result of the calculation indicates that the primary sodium distribution is almost uniform.

The calculated result of one 60-degree sector with heat transfer tubes is shown in Figure 19. It is seen that the flow on the primary side of the IHX is rather uniform in low flow conditions. The next step is a corresponding calculation taking into account the heat transfer between the primary and the secondary side. This work is presently ongoing. 


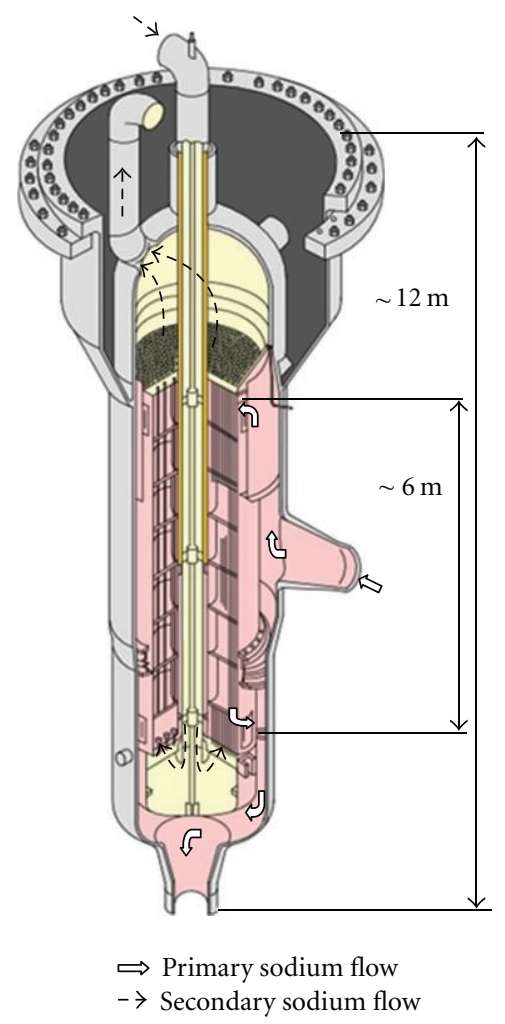

FIgURE 16: Schematic of the IHX of Monju.

4.2. Thermal Stratification Calculation in the Upper Plenum. The analysis of the occurrence of thermal stratification in the upper plenum of the Monju reactor is the subject of a Coordinated Research Program (CRP) organized by the IAEA. Temperature distributions in the upper plenum of the reactor vessel were measured at Monju when the plant was scrammed from a partial load condition during a turbine trip test at $40 \%$ of nominal electrical output ( $45 \%$ of nominal thermal output). The primary heat transport system (HTS) and the secondary HTS were cooled by the forced circulation after the reactor trip with small capacity motors, so-called pony motors. The flow rates in the three primary and secondary HTSs decreased to approximately $1 / 10$ of the full power conditions. This test and its results are summarized in the report by Yoshikawa and Minami [23] as a document of the IAEA CRP.

There are two problems in the IAEA CRP benchmark. One is the total flow rate from the core to the upper plenum. The other problem is configuration of the flow-holes provided on the inner shroud in the reactor vessel. The total flow rate from each group of subassemblies specified in the IAEA benchmark calculation is approximately $97 \%$ of the measured flow rate at the Monju plant. This discrepancy was identified when the test data by Miyakawa [24] was investigated. The outlet temperatures from each group of subassemblies in the IAEA benchmark are measured, except for the shielding subassemblies, for which calculated temperatures are used. Therefore, the boundary conditions for the IAEA benchmark are somewhat hybrid (combination of

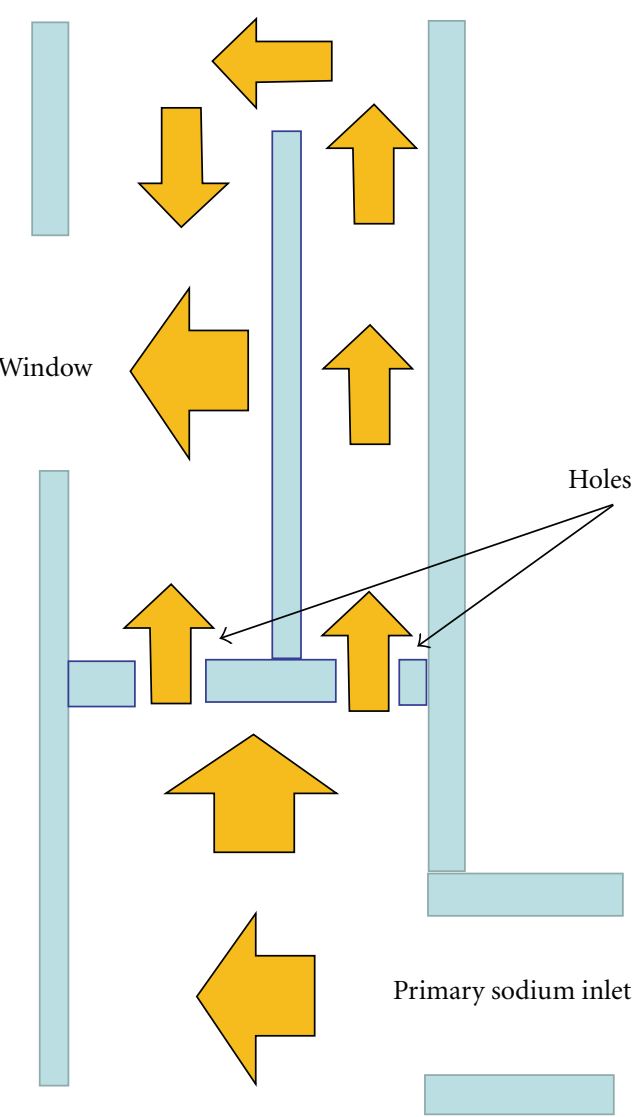

FIGURE 17: Flow distribution mechanism in the annulus of the IHX vessel.

calculated and measured data). The total amount of energy input into the upper plenum is underestimated if the total flow rate does not include the bypass flow rate. There is a possibility that this may be caused by the boundary conditions of flow rate and temperature, which are different from the actual situation.

In order to maintain consistency of the boundary conditions from the point of view of the amount of energy transferred into the upper plenum, a 1D analysis has been conducted using the NETFLOW++ code developed by Mochizuki [25]. NETFLOW++ was used to calculate the outlet flow rates and temperatures of all the assemblies in the core. This data was then used in a 3D CFD analysis of the hot plenum. The calculation was conducted using ANSYS FLUENT 12.0 [26]. The calculational mesh is shown in Figure 20, taking into account almost all internals of the Monju reactor and the configuration of the flow holes. Since the precise roundness of the edges of the flow-holes is not known, a rounded edge with a radius of $20 \mathrm{~mm}$ (R20) is assumed in the present study. The friction factor of a rounded flow hole saturates above a given roundness. Thus, it is expected that the calculated result is not very sensitive to the actual roundness of the flow holes. In earlier work, Mochizuki et al. [27] assumed that the flow-holes had a chamfered configuration. It was shown that the presence of chamfer has a considerable effect on the temperature distribution in the hot plenum. 


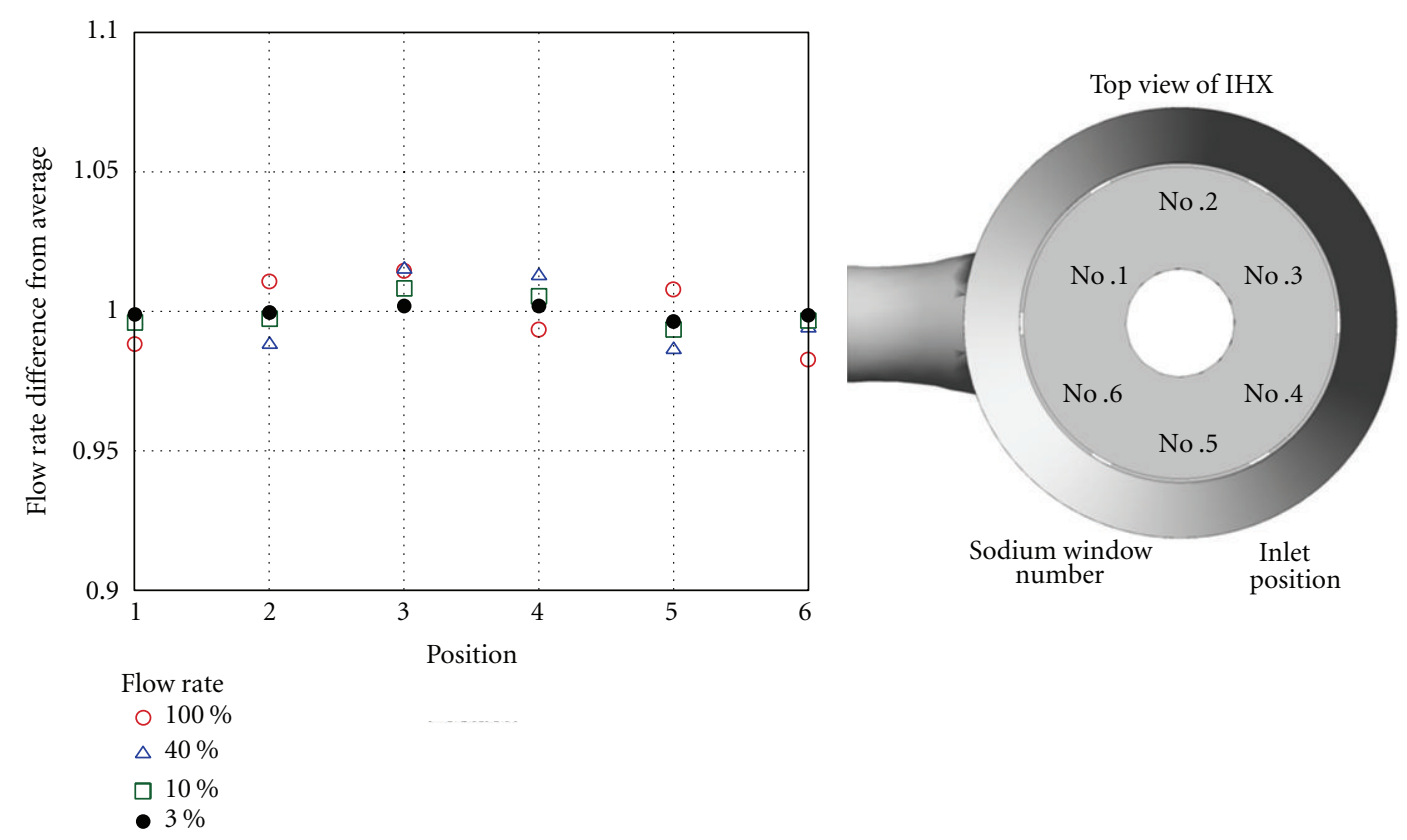

FIgURE 18: Flow rate distribution at the different window positions.

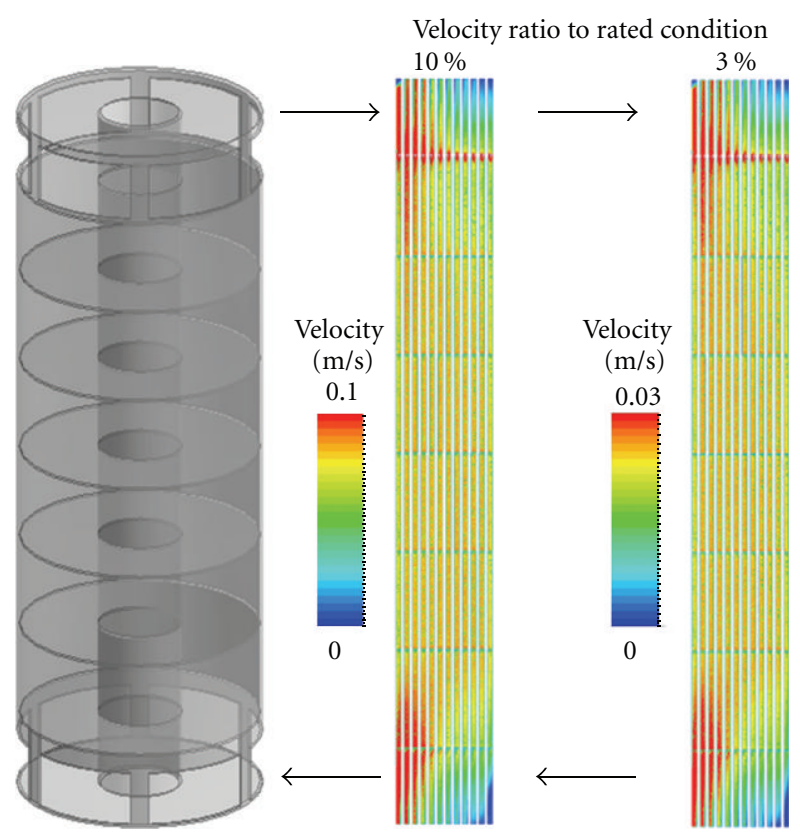

FIGURE 19: Flow pattern on the primary side of the shell of IHX.
Figure 21 illustrates the behavior of the thermal stratification interface during the first 10 minutes of the transient. The temperature is distributed in three layers, that is, a lowtemperature layer, a mixing layer, and a high-temperature layer. Although there are some parts where the temperature distribution does not completely match with the measured result, good agreement has been obtained in general. The thermal stratification interface is defined as the surface between material regions at different temperatures (the low

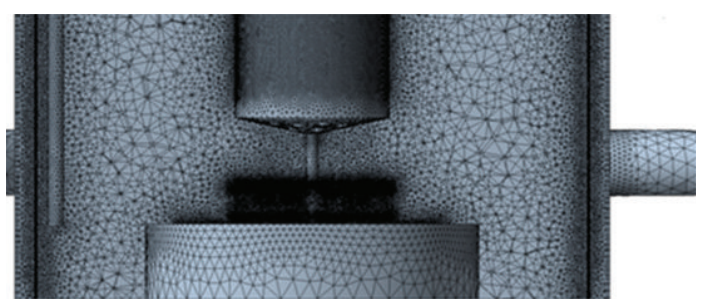

FIGURE 20: Tetrahedral mesh configuration in the plenum (25 million).

TABLE 2: Movement of the thermal stratification interface between measurement and calculation.

\begin{tabular}{lcc}
\hline Elapsed time $(\mathrm{sec})$ & Measured result $(\mathrm{m})$ & Analytical result $(\mathrm{m})$ \\
\hline 60 & -5.38 & -5.43 \\
120 & -4.82 & -4.86 \\
180 & -4.51 & -4.46 \\
240 & -4.37 & -4.32 \\
420 & -4.09 & -4.06 \\
600 & -4.06 & -3.99 \\
\hline
\end{tabular}

temperature area below the interface and the mixing area above the interface). This interface moves up during the transient. A comparison of the elevation of the thermal stratification interface between calculation and measurement is shown in Table 2. As expected from the results in Figure 20, the movement of the thermal stratification interface is well predicted by the CFD model. 


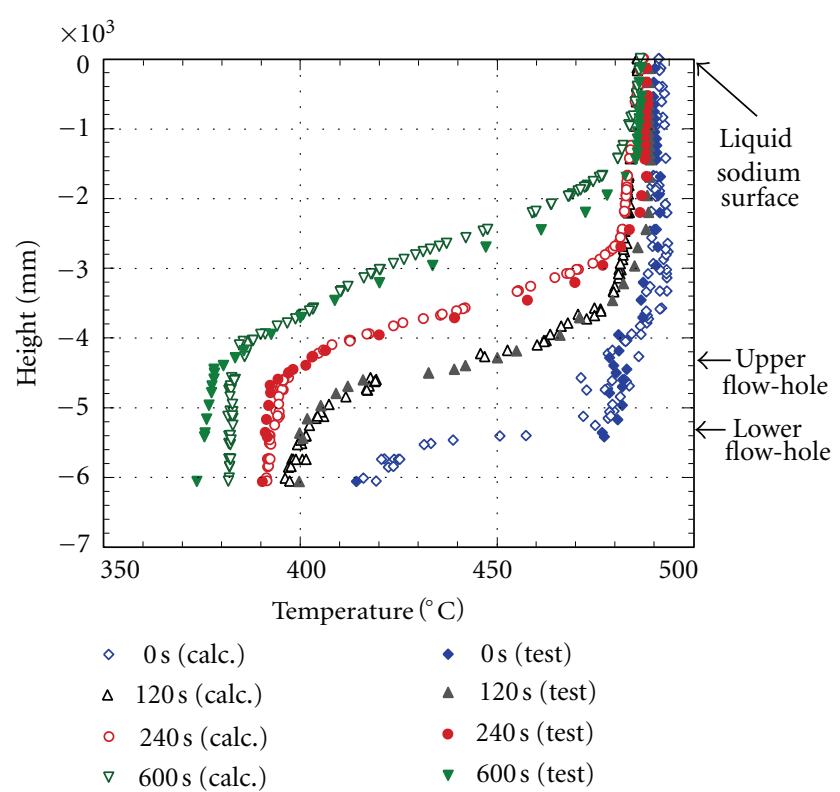

Figure 21: Comparison between measured results and calculation taking into account the flow-hole with rounded edge, heat capacity of UIS, and boundary conditions at the outlet of the subassemblies.

\section{Conclusion}

In this paper, we have given an overview of recent development work on calculational and experimental methods to support the design and analysis of the commercial size (Japanese) Sodium-cooled Fast Reactor (JSFR). This reactor has unique design features, and new methods have been developed to enable highly accurate modeling of JSFR. In the area of neutronics, methods have been devised to treat the complex fuel subassemblies, based on a hyper-fine group calculation for self-shielding and MOC for geometrical treatment. Modeling has been improved for particle dispersion MOX pellets, and for annular pellets, a special annular sensor has been developed to measure the thermal conductivity with a hot disk method. In the area of thermal hydraulics, calculation accuracy of difficult problems, for example, the intermediate heat exchanger and thermal stratification in the hot plenum under low flow conditions, has been improved by the combined use of 1D and 3D CFD models. In all cases, the trade-off between modeling accuracy and practical usability has been optimized. At present, Verification, Validation, and Uncertainty Qualification of all proposed methods is on-going, using measurement data from the prototype FBR Monju.

\section{Acknowledgment}

The presented work is the result of a part of the "Core $R \& D$ program for commercialization of the fast breeder reactor using Monju data," entrusted to the University of Fukui by the Ministry of Education, Culture, Sports, Science and Technology of Japan (MEXT).

\section{References}

[1] K. Aizawa, "R\&D for fast reactor fuel cycle technologies in JNC," in Proceedings of the Advanced Nuclear Fuel Cycles and Systems (GLOBAL '01), vol. 050, Paris, France, September 2001.

[2] K. Ito and T. Yanagisawa, "Last twenty years experiences with fast reactor in Japan," in Proceedings of the International Conference on Fast Reactors and Related Fuel Cycles, Kyoto, Japan, December 2009.

[3] Y. Chikazawa, S. Kotake, and S. Sawada, "Comparison of pool/loop configurations in the JAEA feasibility study 19992006," in Proceedings of the International Conference on Fast Reactors and Related Fuel Cycles, Kyoto, Japan, December 2009.

[4] Y. Sagayama, "Launch of fast reactor cycle technology development project in Japan," in Proceedings of the Advanced Nuclear Fuel Cycles and Systems (GLOBAL '07), pp. 251-258, Boise, Idaho, USA, September 2007.

[5] H. Funasaka and M. Itoh, "Perspective and current status on fuel cycle system of Fast Reactor Cycle Technology development (FaCT) project in Japan," in Proceedings of the Advanced Nuclear Fuel Cycles and Systems (GLOBAL '07), pp. 259-267, Boise, Idaho, USA, September 2007.

[6] K. Aoto, Y. Chikazawa, S. Kotake, and T. Ito, "Status of JSFR development in phase I FaCT project," in Proceedings of the International Conference on Advanced Nuclear Fuel Cycles and Systems (GLOBAL '11), Makuhari, Japan, December 2011, Paper no. 471611.

[7] A. E. Waltar, D. R. Todd, and P. V. Tsvetkov, Fast Spectrum Reactors, Appendix C., Springer, 2011.

[8] G. Chiba, K. Okumura, K. Sugino et al., "JENDL-4.0 benchmarking for fission reactor applications," Journal of Nuclear Science and Technology, vol. 48, no. 2, pp. 172-187, 2011.

[9] T. Hazama, A. Shono, and K. Sugino, "Verification of a nuclear analysis system for fast reactors using BFS-62 critical experiment," Journal of Nuclear Science and Technology, vol. 41, no. 12, pp. 1145-1154, 2004.

[10] T. Hazama, G. Chiba, W. Sato, and K. Numata, "SLAROM-UF: Ultra Fine Group Cell Calculation Code for Fast ReactorVersion 20090113," JAEA-Review 2009-003, 2009.

[11] T. Hazama, G. Chiba, and K. Sugino, "Development of a fine and ultra-fine group cell calculation code SLAROM-UF for fast reactor analyses," Journal of Nuclear Science and Technology, vol. 43, no. 8, pp. 908-918, 2006.

[12] K. Aoto, N. Uto, Y. Sakamoto, T. Ito, M. Toda, and S. Kotake, "Design study and R\&D progress on Japan sodium-cooled fast reactor," Journal of Nuclear Science and Technology, vol. 48, no. 4, pp. 463-471, 2011.

[13] T. Takeda, H. Imai, T. Kitada, H. Nishi, J. Ishibashi, and A. Kitano, "Development of 3-D detailed FBR core calculation method based on method of characteristics," in Proceedings of the International Confernce Mathematics and Computation, Supercomputing, Reactor Physics and Nuclear \& Biological Applications, Avignon, France, September 2005.

[14] K. Sugino and T. Kugo, "Effect of polynomial expansion order of intranode flux treatment in nodal $S_{\mathrm{N}}$ transport calculation code NSHEX for large-size fast power reactor core analysis," Journal of Nuclear Science and Technology, vol. 48, no. 3, pp. 421-428, 2011.

[15] T. Mizuno and S. Shikakura, "Development of the fast reactor fuel performance code CEDAR," PNC Technical Review, no. 76, pp. 37-44, 1990 (Japanese), http://jolissrch-inter.tokai-sc.jaea.go.jp/pdfdata/PNC-TN1340-90-004.pdf. 
[16] A. B. G. Washington, "UK atomic energy authority," Tech. Rep. TRG R-2236, 1973.

[17] Y. Philipponneau, "Thermal conductivity of $(\mathrm{U}, \mathrm{Pu}) \mathrm{O}_{2-\mathrm{x}}$ mixed oxide fuel," Journal of Nuclear Materials, vol. 188, pp. 194-197, 1992.

[18] C. Duriez, J. P. Alessandri, T. Gervais, and Y. Philipponneau, "Thermal conductivity of hypostoichiometric low Pu content (U,Pu) $\mathrm{O}_{2-x}$ mixed oxide," Journal of Nuclear Materials, vol. 277, no. 2-3, pp. 143-158, 2000.

[19] K. Iwasaki, T. Matsui, K. Yanai et al., "Effect of $\mathrm{Gd}_{2} \mathrm{O}_{3}$ dispersion on the thermal conductivity of $\mathrm{UO}_{2}$," Journal of $\mathrm{Nu}$ clear Science and Technology, vol. 46, no. 7, pp. 673-676, 2009.

[20] S. Humphries, Field Solutions on Computers, CRC Press, Boca Raton, Mass, USA, 1997.

[21] S. E. Gustavsson, "Transient plane source techniques for thermal conductivity and thermal diffusivity measurements of solid materials," Review of Scientific Instruments, vol. 62, no. 3, pp. 797-804, 1991.

[22] R. W. Rice, Porosity of Ceramics, Marcel Dekker, New York, NY, USA, 1998.

[23] S. Yoshikawa and M. Minami, Data Description for Coordinated Research Project on Benchmark Analyses of Sodium Natural Convection in the Upper Plenum of the Monju Reactor Vessel under Supervisory of Technical Working Group on Fast Reactors, International Atomic Energy Agency, Japan Atomic Energy Agency, 2008.

[24] A. Miyakawa, "System start up test of the prototype fast breeder reactor MONJU," JAEAR\&D Review, p. 21, 2006.

[25] H. Mochizuki, "Development of the plant dynamics analysis code NETFLOW++," Nuclear Engineering and Design, vol. 240, no. 3, pp. 577-587, 2010.

[26] Ansys Inc., Ansys Fluent 12.0 User’s Guide, 2009.

[27] H. Mochizuki, M. Takano, and H. Yao, "Analysis of thermal stratification in the upper plenum of the "Monju" reactor vessel-effect of chamfer of flow hole on thermal stratification," in Proceedings of the 14th International Topical Meeting on Nuclear Reactor Thermalhydraulics (NURETH '11), Toronto, Canada, 2011. 

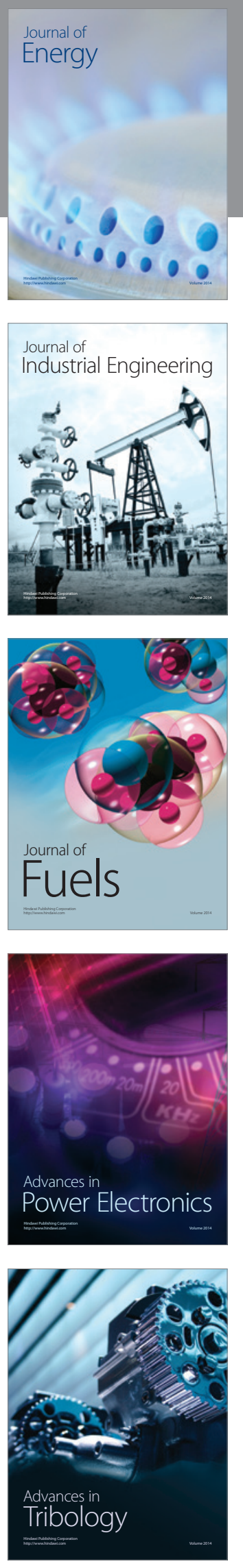
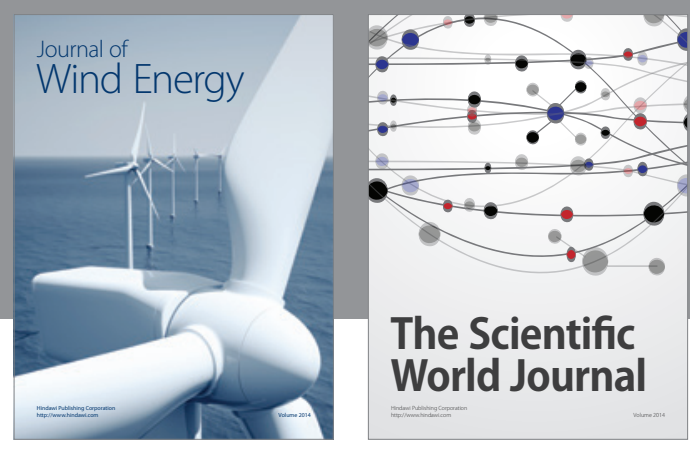

The Scientific World Journal

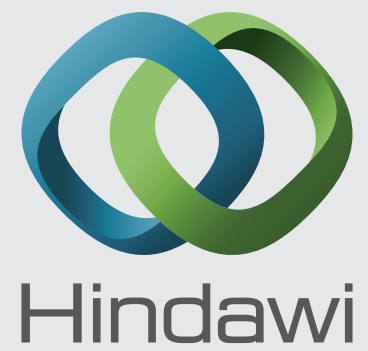

Submit your manuscripts at http://www.hindawi.com
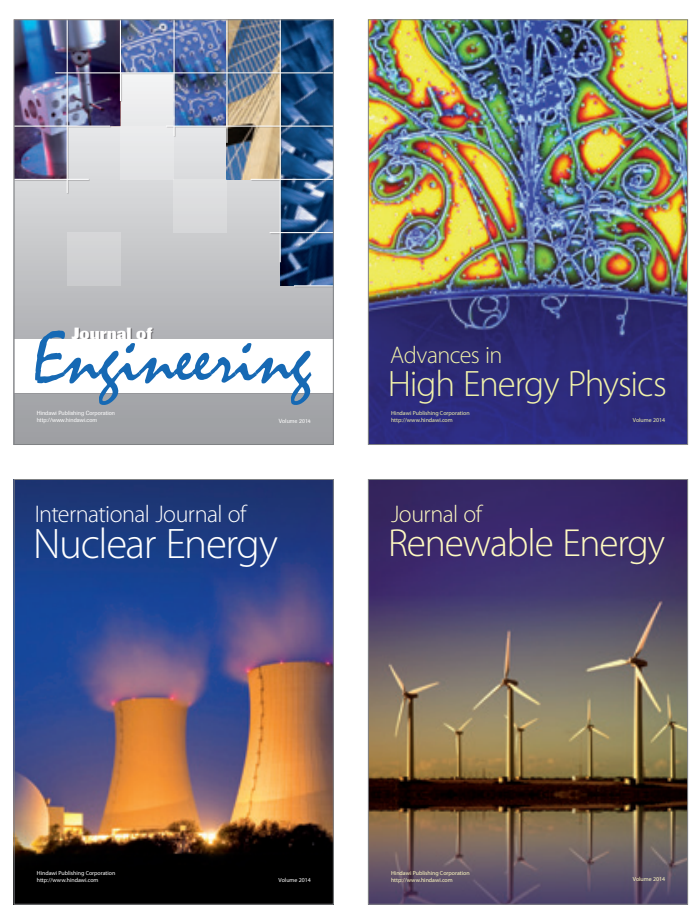

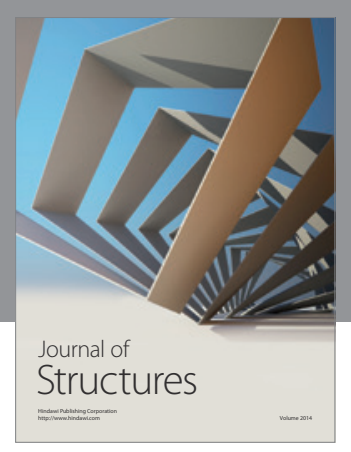

Rotating
Mechinery
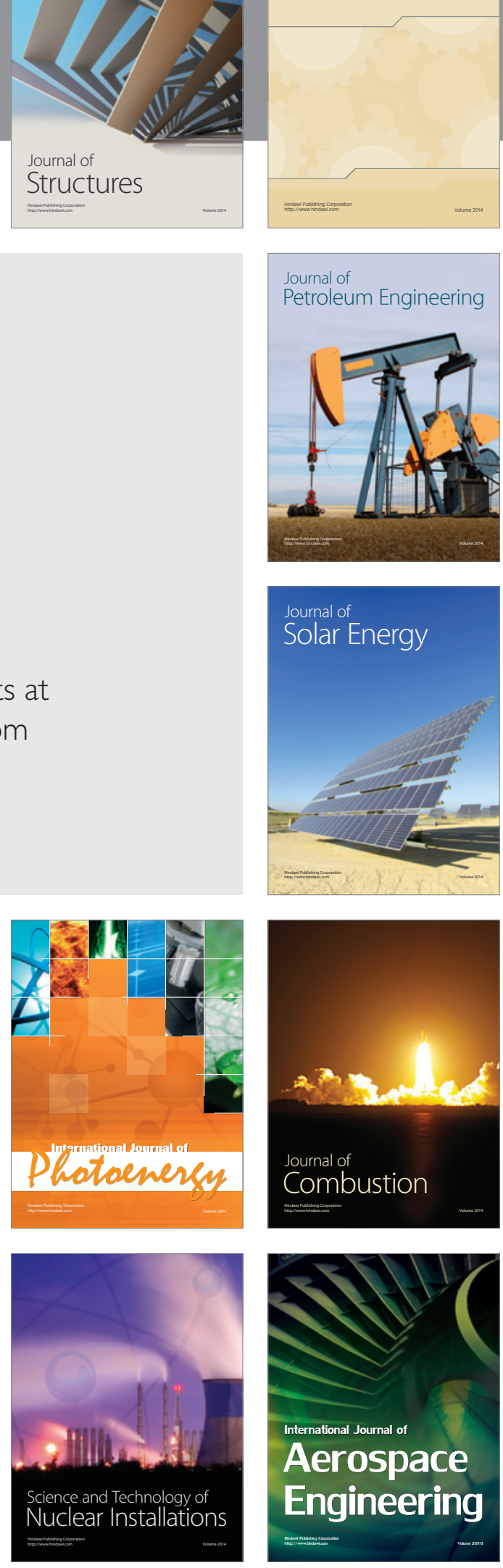